\title{
Analysis of Babesia bovis infection-induced gene expression changes in larvae from the cattle tick, Rhipicephalus (Boophilus) microplus
}

Andrew M Heekin ${ }^{1 *}$, Felix D Guerrero ${ }^{1}$, Kylie G Bendele ${ }^{1}$, Leo Saldivarr ${ }^{2}$, Glen A Scoles ${ }^{3}$, Cedric Gondro ${ }^{4}$, Vishvanath Nene ${ }^{5}$, Appolinaire Djikeng ${ }^{5}$ and Kelly A Brayton ${ }^{6}$

\begin{abstract}
Background: Cattle babesiosis is a tick-borne disease of cattle that has severe economic impact on cattle producers throughout the world's tropical and subtropical countries. The most severe form of the disease is caused by the apicomplexan, Babesia bovis, and transmitted to cattle through the bite of infected cattle ticks of the genus Rhipicephalus, with the most prevalent species being Rhipicephalus (Boophilus) microplus. We studied the reaction of the $R$. microplus larval transcriptome in response to infection by B. bovis.

Methods: Total RNA was isolated for both uninfected and Babesia bovis-infected larval samples. Subtracted libraries were prepared by subtracting the B. bovis-infected material with the uninfected material, thus enriching for expressed genes in the B. bovis-infected sample. Expressed sequence tags from the subtracted library were generated, assembled, and sequenced. To complement the subtracted library method, differential transcript expression between samples was also measured using custom high-density microarrays. The microarray probes were fabricated using oligonucleotides derived from the Bmi Gene Index database (Version 2). Array results were verified for three target genes by real-time PCR.

Results: Ticks were allowed to feed on a B. bovis-infected splenectomized calf and on an uninfected control calf. RNA was purified in duplicate from whole larvae and subtracted CDNA libraries were synthesized from Babesiainfected larval RNA, subtracting with the corresponding uninfected larval RNA. One thousand ESTs were sequenced from the larval library and the transcripts were annotated. We used a $R$. microplus microarray designed from a $R$. microplus gene index, BmiGl Version 2, to look for changes in gene expression that were associated with infection of $R$. microplus larvae. We found 24 transcripts were expressed at a statistically significant higher level in ticks feeding upon a B. bovis-infected calf contrasted to ticks feeding on an uninfected calf. Six transcripts were expressed at a statistically significant lower level in ticks feeding upon a B. bovis-infected calf contrasted to ticks feeding on an uninfected calf.
\end{abstract}

\footnotetext{
* Correspondence: Andrew.Heekin@ars.usda.gov

'USDA-ARS, Knipling Bushland US Livestock Insect Research Laboratory, 2700 Fredericksburg Rd, Kerrville, TX 78028, USA

Full list of author information is available at the end of the article
} 
Conclusion: Our experimental approaches yielded specific differential gene expression associated with the infection of R. microplus by B. bovis. Overall, an unexpectedly low number of transcripts were found to be differentially expressed in response to B. bovis infection. Although the BmiGl Version 2 gene index (http://compbio. dfci.harvard.edu/tgi/cgi-bin/tgi/gimain.pl?gudb=b_microplus) was a useful database to help assign putative function to some transcripts, a majority of the differentially expressed transcripts did not have annotation that was useful for assignment of function and specialized bioinformatic approaches were necessary to increase the information from these transcriptome experiments.

Keywords: Cattle tick, Rhipicephalus (Boophilus) microplus, Babesia bovis, Larva, Transcriptome, Serine proteinase inhibitor

\section{Background}

The cattle tick, Rhipicephalus (Boophilus) microplus, is a one-host tick parasitizing cattle in most of the world's tropical and subtropical countries. This tick has a huge impact on cattle producers, large and small, with losses due to $R$. microplus infestations in Brazil alone estimated to be over $\$ 2$ billion annually [1]. Perhaps the major impact is due to losses attributable to pathogens and their associated diseases transmitted by the tick's bite. $R$. microplus is known to frequently harbor Anaplasma marginale, the causative agent of anaplasmosis, and Babesia bovis and Babesia bigemina, the apicomplexan agents that cause cattle babesiosis. The cattle- $R$. microplus-Babesia complex has been described as the most important agricultural host-arthropod-pathogen complex globally [2]. B. bovis is generally responsible for the more serious cases of bovine babesiosis, with infection of naive hosts often causing pulmonary edema, central nervous system problems, and death. The severity of this disease in Australia is such that an anti-B. bovis vaccine is in widespread use across northern Australia in response to seasonal outbreaks in the major cattle producing areas [3].

When R. microplus ingests blood from a B. bovisinfected animal, the ingested merozoites undergo developmental changes in the tick midgut until the zygote stage of the apicomplexan enters the digestive cells of the tick's gut where further multiplication and development to the kinete stage occurs. This kinete stage eventually enters the hemolymph and spreads to the rest of the tick's tissues [3]. We are interested in transcriptional changes that accompany the various stages of infection by $B$. bovis as it interacts with its tick host. As our biological system, we allowed $R$. microplus to feed upon a splenectomized calf suffering from bovine babesiosis due to infection with $B$. bovis. We compared gene expression in larvae that hatched from eggs oviposited by adult female ticks that had fed on this infected calf with gene expression in larvae that hatched from eggs oviposited by adult female ticks that had fed on an uninfected calf. Our approaches included sequencing subtracted libraries synthesized from infected larvae, microarray analysis, and quantitative real-time polymerase chain reaction (qRT-PCR) to identify and annotate transcripts associated with $B$. bovis infection of $R$. microplus larvae.

\section{Methods}

Ticks

$R$. microplus larvae were from the 16 generation of the B. bovis-free La Minita strain. The La Minita strain was started from engorged female ticks collected from an outbreak in Starr County, Texas in 1996 and propagated at the USDA Cattle Fever Tick Research Laboratory at Moore Field, Texas before being transferred to the USDA-ARS Animal Disease Research Unit (ADRU) in Pullman, WA. Two splenectomized Holstein calves, 5-6 months of age, were used in these studies. One of the calves was infected by inoculating with frozen blood stabilate of the Texas Strain ( $2^{\text {nd }}$ passage), of B. bovis. The infection status of this calf was verified by blood testing and subsequently used to provide $B$. bovis infected tick larvae for this study. The second calf was maintained infection-free and was used to provide uninfected tick larvae for this study. All animal use was conducted at ADRU facilities at the University of Idaho Holm Research Center (Moscow, ID) while following protocols approved by the University of Idaho Institutional Animal Care and Use Committee.

$B$. bovis-infected larvae were obtained by placing uninfected larvae that hatched from $1.0 \mathrm{~g}$ of eggs from the $B$. bovis-free La Minita colony ticks into feeding patches glued to the shaved back and sides of the B. bovisinfected calf (designated as day 1). After feeding, all larvae were removed before they molted to the nymphal stage and frozen at $-80^{\circ} \mathrm{C}$ immediately.

Uninfected larvae were obtained by placing uninfected larvae that hatched from $1.0 \mathrm{~g}$ of eggs from the B. bovisfree La Minita colony ticks into feeding patches glued to the shaved back and sides of the infection-free calf (designated as day 1). After feeding, all larvae were removed before they molted to the nymphal stage and frozen at $-80^{\circ} \mathrm{C}$ immediately. 


\section{RNA protocols}

Separately, for both the uninfected and Babesia bovisinfected larval samples, total RNA was isolated from a pool of several thousand tick larvae using the FastPrep-24 Tissue and Cell Homogenizer and Lysing Matrix D (Qbiogene, Irvine, CA, USA) as described in Saldivar et al. (2008) [4]. Total RNA was treated with Turbo DNAse per Turbo DNA-free kit protocols (Ambion Inc.). RNA integrity was verified by formaldehyde gel electrophoresis and staining in GelStar Nucleic Acid Gel Stain (Lonza, Rockland, ME, USA). Twenty $\mu$ g of DNA-free total RNA was sent to NimbleGen Systems Inc. (Madison, WI, USA) for use in microarray hybridization. Express Genomics Inc. (Frederick, MD, USA) was used to produce subtracted libraries from $0.15 \mathrm{mg}$ each of $B$. bovis-infected and uninfected larval total RNA. The subtracted libraries, produced by subtracting the $B$. bovis-infected material with the uninfected material, were enriched for expressed genes in the $B$. bovis-infected sample.

\section{Sequencing and bioinformatics}

Library sequencing was performed at the J. Craig Venter Institute (Rockville, MD). Bacterial colonies were picked for template preparation using colony-picking robots (Genetix, Boston, MA), inoculated into 384 well plates containing liquid medium and grown overnight. A robotic workstation was used to prepare sequencing grade plasmid DNA using an alkaline lysis method modified for high throughput processing [5]. Beckman Multimek 96 or Biomek FX automated pipetting robot work stations (Beckman Coulter, Fullerton, CA) were used to combine pre-aliquoted templates and reaction mixes consisting of deoxy- and fluorescently labeled dideoxynucleotides, Taq DNA polymerase, sequencing primers, and sequencing reaction buffer. Linear amplification steps were performed on MJ Research Tetrads PTC-225 (MJ Research, Inc., Watertown, MA) and sequencing reaction products purified by ethanol precipitation and resolved on ABI 3730xl sequencing machines (Applied Biosystems, Foster City, CA).

The larval subtracted library expressed sequence tags (ESTs) were assembled into 469 contigs with Paracel Transcript Assembler (2002) with the default assembly parameters (http://www.paracel.com/), and submitted to GenBank TSA with accession numbers JT844344JT844812. The remaining 306 singleton reads, representing paired-end sequences that did not assemble, were submitted to GenBank dbEST with the accession numbers of FG579553-FG579858. All ESTs were screened for vector contamination with the SeqClean vector trimming utility downloaded from the Dana Farber Institute (http://compbio.dfci.harvard.edu/tgi/software/). Multiple sequence alignments were performed with the online tool PRRN using the default settings except for the gap open penalty, which was lowered to 7.0 to achieve better scoring alignments (http://www.genome.jp/tools/prrn/). To determine presence of rRNA in the subtracted library, a blastn search was performed against a eukaryotic rRNA database assembled from the European ribosomal RNA database (http://www.psb.ugent.be/ rRNA/) with the recommended expect value ( $e$-value) cutoff of 1e-65 [6]. Samples were similarly screened for mitochondrial RNA by searching a database of mitochondrial proteins curated by the National Center for Biotechnology and Information (NCBI) using the $e$-value cutoff of 1e-08 (ftp://ftp.ncbi.nlm.nih.gov/blast/db/FASTA/ mito.aa.gz). All preprocessed EST contigs and singletons less than 200 base pairs in length were discarded. The term "unigene" will be used to refer to a contig or a singleton throughout this study.

\section{EST annotation}

Annotations were assigned to ESTs in this study in three stages. Similarity search methods of extant protein databases generally produce more accurate annotations than de novo prediction methods [6]. We therefore annotated sequences using similarity searches of the Uniref100 database as the first stage. UniRef100 contains all the records in the UniProt knowledgebase and merges identical sequences and subfragments as a single entry, and thus increases speed and accuracy of homology searches [7]. Homology searches of Uniref100 were performed with the BLAST tool BLASTX [8], which translates the query to all 6 possible reading frames using an $e$-value cutoff of 1e-07. Sequences with no high-scoring pairs (HSPs) from the Uniref100 BLAST search were passed to the second annotation stage.

The second stage analysis was performed with two open-source software platforms: annot8r and prot4EST. Detecting the correct reading frame for each EST is essential for robust de novo function prediction. The prot4EST software package includes a pipeline to correct EST datasets for frame shifts [6]. Sequence data were run through the prot4EST pipeline prior to annotation. The annot8r package is a tool for assignment of Gene Ontology (GO), Enzyme Commission (EC) and Kyoto Encyclopaedia of Genes and Genomes (KEGG) annotations [9]. GO constitutes a controlled vocabulary to describe function and location of gene products (The Gene Ontology Consortium, 2000). EC is a hierarchical enzyme classification based on the type of reaction catalyzed [10]. KEGG annotates biochemical pathways for wholly sequenced genomes [11]. In a preprocessing step, annot8r automatically downloads relevant files and generates a reference database that stores UniRef100 entries with GO, EC and KEGG annotations. For each of the GO, EC and KEGG entries, annot8r extracts a specific sequence subset from the UniRef dataset based on 
matches to the reference database. These three subsets are then formatted for BLAST searches. To annotate sequence data, annot8r conducts BLASTX searches with the recommended minimum cutoff bit score of 55 against these three subsets. The BLAST results are then parsed and the corresponding annotations retrieved from the reference database.

In the final stage of our analysis we used InterProScan, which is a software package that integrates the common methodologies in the area of protein family, domain and motif detection. The InterProScan package identifies signatures from the InterPro member databases by applying disparate algorithms [12]; see Additional file 1 for a complete listing of available databases and applicable analysis tools. This additional annotation was attempted on each EST regardless of whether it appeared in the first or second stage BLAST searches, and therefore allowed the identification of more distant evolutionary relationships.

To quantify the effect of B. bovis on infected larvae, we focused on unigenes that were annotated with one of three terms from the GO ontology. The GO term "immune response" (GO:0006955) includes both innate and adaptive immune responses. The GO term "stress response" (GO:0006950) is defined as an exogenous change in state or activity of a cell or an organism as a result of a disturbance in its homeostasis. The GO term "defense response" (GO:0006952) is a specific (child) term of stress response defined as a triggered response to the presence of a foreign body or the occurrence of an injury; it also includes some responses of the innate immune system.

\section{Microarray design and analysis}

Custom high-density single channel oligonucleotide arrays were constructed by NimbleGen Systems Inc. using 13,601 of the 13,642 members of BmiGI Version 2 and 14 perfect-match 50 -mer probes per BmiGI target; these microarrays are described in detail by Saldivar et al. [4]. Probes with randomly generated sequences were designed into the arrays, but no mismatch probes were included. Each array chip includes two in-slide replicates, called spot replicates which have the same probe spotted on different locations on the chip, and are considered technical replicates as each of the probes for the 13,601 Gene Index members are spotted on different locations within the chip. The spot replicates increase precision and provide a basis for testing differences within treatment groups. Because of the status of $R$. microplus as an arthropod requiring adherence to strict United States Department of Agriculture (USDA) quarantine and handling restrictions and because of the need to sacrifice the calves at the end of each experiment, the ideal independent biological replicates were not available.
Instead, we utilized pooled tick samples sourcing from an experiment with a single calf for each of the infected and uninfected feeding experiments. Our array experimental design consisted of four technical replicates, i.e. repeated measurements of the same $R$. microplus mRNA isolated from the feeding larval sample recovered and pooled from the single B. bovis infected or uninfected calf. In total, the design consisted of two chip replicates, each chip containing two spot replicates that are located on each chip. Samples were labeled before hybridization to the microarrays. After scanning the arrays, image analysis was conducted at NimbleGen Systems Inc. as described by Saldivar et al. [4]. Quality control measures and preprocessing were performed using the statistical computing language $\mathrm{R}$ and Bioconductor [13,14]. All microarray images and quality control measurements were within recommended limits, although one of the two replicate larval arrays was marginal. The quality of the arrays was assessed through standard quality control measures including: pseudo-images of the arrays (to detect spatial effects), scatter plots of the arrays versus a pseudo-median reference chip, and additional summary statistics including histogram, box plots of raw and normalized log intensities.

The intensity raw values were normalized using quintile normalization, gene calls generated using the Robust Multichip Average (RMA) algorithm [15,16], and raw intensity data $\log$ base $2(\log 2)$ transformations [4]. The microarray datasets have been submitted to the GEO database (www.ncbi.nlm.nih.gov/geo/; GEO accession number GSE10816). The data files were loaded into Microarray Experiment Viewer (MeV Version 4.0, DanaFarber Cancer Institute, Boston, MA, USA) and Significance Analysis of Microarrays (SAM) [17,18] selected statistically significant differentially expressed genes. A threshold value delta equal to 1.8 and a fold change $\geq 2.0$ were used to separate significant from non-significantly differentially expressed ESTs. With the selected delta and fold change parameters, SAM estimated the proportion of false positives as $<0.00001$.

\section{Verification by Real-Time PCR}

Array results were selected for verification based on their level of differential expression and the amount of annotation available for their corresponding BmiGI sequence. The same total RNA samples used for the microarrays were also used for the real-time PCRs. Four micrograms of DNA-free total RNA for each sample was used according to manufacturer's recommendations with the RETROscript Kit Reverse Transcription for RT-PCR (Ambion) to produce cDNA for each sample. Primers and TaqMan probes were designed using Beacon Designer 7.5 (PREMIER BioSoft International, Palo Alto, CA; Table 1) and synthesized by Sigma-Aldrich Inc. 
(Atlanta, GA) for each EST selected and for the $R$. microplus 18S rRNA gene, which was used as reference gene for normalization [4]. Validation and primer optimization experiments were run on each EST and reference gene to determine the efficiencies of the target ESTs and reference gene and optimal concentrations to be used in individual experiments. All real-time reactions were carried out in clear low 96 well plates (no. MLL9601, BioRad, Hercules, CA) and sealed with Microseal B film (BioRad) using $25 \mu \mathrm{L}$ total reaction volumes including primers, 250nM TaqMan probe, TaqMan Universal Master Mix No AmpErase UNG (Applied Biosystems Inc., Foster City, CA) and corresponding RETROscript cDNA. The final primer concentration for the 18S rRNA reference gene and the ESTs were $900 \mathrm{nM}$ for both the forward and reverse primers with the exception of 300nM for the TC9020 reverse primer. All primer and probe sequences are listed in Table 1. The BioRad CFX96 Real-Time System was used with a cycling protocol of $95^{\circ} \mathrm{C}$ for 10 minutes, 50 cycles of $95^{\circ} \mathrm{C}$ for 15 seconds, and $60^{\circ} \mathrm{C}$ for 1 minute plus plate read. The fluorescence emission data analysis was done using the baseline subtracted curve fit mode with CFX Manager Software version 1.0 (BioRad).

\section{Results and discussion}

We studied differential gene expression in $R$. microplus associated with $B$. bovis infection to better understand the interplay between the larval life stage of the tick host and the invading apicomplexan pathogen as the infection process initially takes place. We infected tick larvae by allowing $B$. bovis-free larvae to feed upon a B. bovisinfected calf and for comparison, we repeated the protocol, allowing uninfected larvae to feed upon an uninfected calf. We used various analytical approaches to characterize infection-induced differential gene expression in these larvae.

\section{Microarray results}

From the microarray experiments, 24 transcripts were expressed in larval tissues at a statistically significant (adjusted p-value <0.01) higher level in ticks feeding upon a $B$. bovis-infected calf contrasted to ticks feeding on an uninfected calf (Additional file 2). Six transcripts were expressed in larval tissues at a statistically significant (adjusted p-value <0.01) lower level in ticks feeding upon a $B$. bovis-infected calf contrasted to ticks feeding on an uninfected calf (Additional file 2). Tables 2 and 3 show the greatest up- and down-regulated transcripts, respectively, with fold-change and annotation data. As similarly reported by Saldivar et al. [4], a number of the significantly differentially expressed genes have no useful annotation. Three of the 20 transcripts in Table 2 and 4 of the transcripts in Table 3 lacked significant ( $e$-value $<$ 0.001) BLASTX hits.

We attempted to identify genes whose transcripts played significant roles in the host-pathogen interactions between $B$. bovis and $R$. microplus. Our microarray approach identified infection-associated transcripts from a preexisting EST database, BmiGI Version 2.0. Interestingly, the second greatest up-regulated transcript in the larval microarrays, TC11482, had sequence similarity to cytochrome P-450, a family of genes whose products are often involved in a rapid response to external environmental stresses, including detoxification of xenobiotics (Table 2). The unigene TC9012 was also up-regulated in the microarrays and shared sequence similarity to glutathione S-transferase, another gene family often involved in detoxification or stress response. In fact, TC9012 has significant sequence similarity to the glutathione Stransferase DvGST2, which was reported as differentially up-regulated in Dermacentor variabilis in response to infection by Rickettsia montanensis [19]. DvGST2 was also reported as up-regulated upon blood-feeding in the same tick species [20]. The glutathione S-transferase

Table 1 Relative quantitative real-time PCR primers and probes

\begin{tabular}{lll}
\hline EST & Primers & Taqman Probe \\
\hline TC293 & FW 5' AACATCTACAGCAAGTTTGACACC 3' & 5' FAM- AGGTGACCGCCTGGATACTCCGCA-TAMRA 3' \\
BEAl101TR & RV 5' CCCGTCGATGCAGGTTTGG 3' & \\
& FW 5' CGGAAGAAACGAGAAATACGAGAC 3' & 5' FAM- TGCGTCGGCACACACTGGTACAGC-TAMRA 3' \\
TC12256 & RV 5' TACATGAGAACAGTAGCATATAGGG 3' & \\
& FW 5' CTTCACATTCAACACGCCCTAC 3' & 5' FAM-AGCCACAGCAACGCCATCGCCG-TAMRA 3' \\
TC14244 & RV 5' AAAACCGCTACGGCAAATGC 3' & \\
& FW 5' TTAAACATTCTTTCGCTCATCAGTC 3' & 5' FAM-CCGCACGACGCAAGCCGAAA-TAMRA 3' \\
18S & RV 5' TACATGAGAACAGTAGCATATAGGG 3' \\
& FW 5' CCTGAGAAACGGCTACCACATC 3' & \\
\hline
\end{tabular}

\footnotetext{
a Based on BmiGI Version 2 designations (http://compbio.dfci.harvard.edu/tgi/cgi-bin/tgi/gimain.pl?gudb=b_microplus).
}

${ }^{b}$ Forward (FW) \& reverse (RV). 
Table 2 R. microplus microarray transcripts with highest statistically significant up-regulation associated with $B$. bovis infection

\begin{tabular}{|c|c|c|c|c|c|c|}
\hline \multirow[t]{2}{*}{$\mathrm{ID}^{\mathrm{a}}$} & \multirow[t]{2}{*}{$d^{b}$} & \multirow[t]{2}{*}{$\mathrm{FC}^{\mathrm{c}}$} & \multicolumn{4}{|l|}{ BLASTX Annotation } \\
\hline & & & Protein & Species & Accession Number & e-Value \\
\hline TC13643 & 7.3 & 5.0 & Dihydrodipicolinate synthetase & Bradyrhizobium sp. & CCD90265.1 & $3 e-07$ \\
\hline TC11482 & 6.9 & 3.8 & Cytochrome P450-like & Phillyrea latifolia & CAK18871.1 & $9 e-19$ \\
\hline TC5802 & 5.9 & 3.9 & GGY domain protein & Argas monolakensis & ABI52689.1 & $3 e-26$ \\
\hline TC9132 & 5.6 & 2.7 & Hypothetical protein LELG_02536 & Lodderomyces elongisporus & XР001525979.1 & $1 e-04$ \\
\hline TC12256 & 5.6 & 7.6 & Hemelipoglycoprotein precursor & Dermacentor variabilis & ABD83654.1 & 0.0 \\
\hline TC9012 & 5.3 & 3.7 & Glutathione S-transferase & Dermacentor variabilis & ABB46494.1 & $3 e-62$ \\
\hline TC15029 & 4.9 & 7.8 & Hemelipoglycoprotein precursor & Dermacentor variabilis & ABD83654.1 & $1 e-106$ \\
\hline TC9597 & 4.8 & 2.7 & Esterase & Ixodes scapularis & XP002411693.1 & $2 e-58$ \\
\hline TC8343 & 4.8 & 3.2 & Methyltransferase-like 7A & Macaca mulatta & NP001180640.1 & $2 \mathrm{e}-33$ \\
\hline TC8407 & 4.8 & 3.8 & Acyl-CoA synthetase & Ixodes scapularis & ХP002433879.1 & $7 e-62$ \\
\hline TC13794 & 4.7 & 2.2 & $\mathrm{NSS}^{\mathrm{d}}$ & - & - & - \\
\hline BEADQ11TR & 4.5 & 3.3 & Non-LTR retrotransposon & Bombyx mori & BAD82945 & $2 e-24$ \\
\hline TC9454 & 4.5 & 5.0 & Midgut cysteine proteinase 4 & Rhipicephalus appendiculatus & AAO60047.1 & 0.0 \\
\hline TC5904 & 4.5 & 2.1 & NSS & - & - & - \\
\hline TC5872 & 4.5 & 2.9 & GGY domain protein & Argas monolakensis & ABI52689.1 & $2 \mathrm{e}-12$ \\
\hline TC10734 & 4.5 & 4.7 & NSS & - & - & - \\
\hline TC6802 & 4.4 & 2.1 & Carboxypeptidase A2 precursor & Ixodes scapularis & XP002406597.1 & $2 e-96$ \\
\hline BEACP61TR & 4.3 & 2.8 & Microplusin & Rhipicephalus microplus & Q86LE5.1 & $2 \mathrm{e}-69$ \\
\hline TC12142 & 4.2 & 3.2 & Glycine-rich secreted protein & Ixodes scapularis & XP002411976.1 & $1 e-10$ \\
\hline TC12248 & 4.1 & 3.0 & Mucin 68D & Drosophila melanogaster & NP648504.2 & $5 e-07$ \\
\hline
\end{tabular}

${ }^{a}$ The identification number from BmiGl Version 2 (http://compbio.dfci.harvard.edu/tgi/cgi-bin/tgi/gimain.pl?gudb=b_microplus).

${ }^{b} \mathrm{~d}$ is the $\mathrm{d}$ statistic as performed by SAM.

${ }^{\mathrm{C}} \mathrm{FC}$ is the fold change ratio.

${ }^{\mathrm{d}}$ NSS indicates no statistically significant similarity found in BLASTX search, based on $e$-value $<0.001$.

induction, and those of the P-450 transcript discussed above, may be in response to toxic heme by-products of bloodfeeding, rather than to B. bovis infection. However, the uninfected sample controls were at similar developmental stages and actively ingesting blood. Indeed, the heme-induced responses should have been cancelled out in the comparison between control and infected samples. The B. bovis infection is likely imposing unique stresses within the ticks that are responsible for these detoxification responses being induced in a tissuespecific manner.

A small number of differentially expressed transcripts were observed when contrasted with other microarray studies using the same BmiGI Version 2-derived arrays. We

Table 3 R. microplus microarray transcripts with highest statistically significant down-regulation associated with $B$.

bovis

\begin{tabular}{|c|c|c|c|c|c|c|}
\hline \multirow[t]{2}{*}{$\mathrm{ID}^{\mathrm{a}}$} & \multirow[t]{2}{*}{$d^{b}$} & \multirow[t]{2}{*}{$F^{c}$} & \multicolumn{4}{|l|}{ BLASTX Annotation } \\
\hline & & & Protein & Species & Accession Number & e-Value \\
\hline TC14933 & -6.1 & -2.6 & Phosphatidylinositol 4-kinase & Brassica napus & AAQ24839.1 & $1 e-06$ \\
\hline BEAC172TR & -5.3 & -4.6 & $\mathrm{NSS}^{\mathrm{d}}$ & - & - & - \\
\hline TC14244 & -4.9 & -3.4 & NSS & - & - & - \\
\hline TC12338 & -4.7 & -2.2 & NSS & - & - & - \\
\hline BEAC711TR & -4.5 & -2.9 & NSS & - & - & - \\
\hline BEADW01TF & -4.4 & -3.5 & NSS & - & - & - \\
\hline
\end{tabular}

${ }^{a}$ The identification number from BmiGI Version 2 (http://compbio.dfci.harvard.edu/tgi/cgi-bin/tgi/gimain.pl?gudb=b_microplus).

${ }^{b} \mathrm{~d}$ is the $\mathrm{d}$ statistic as performed by SAM.

${ }^{c} \mathrm{FC}$ is the fold change ratio.

${ }^{\mathrm{d}}$ NSS indicates no statistically significant similarity found in BLASTX search, based on e-value $<0.001$. 
Table 4 GO annotation summary by domain

Cellular component (C)

GO ID

\section{Description}

Membrane

GO:0016020

GO:0005623

GO:0005576

GO:0005622

Molecular function (F)

\section{GO ID}

GO:0003774

GO:0016874

GO:0016829

GO:0004871

GO:0016491

GO:0016853

GO:0030234

GO:0003824

GO:0005488

GO:0016740

GO:0005198

GO:0005215

Biological process $(\mathrm{P})$

GO ID

GO:0006944

GO:0050896

GO:0007610

GO:0006810

GO:0030154

GO:0008152

GO:0050789

GO:0043062

GO:0007275

GO:0009987

GO:0007154

GO:0008219

GO:0006139

\section{Description}

Lyase activity

Binding

Description

Behavior

Transport

Cell death

\section{Cell \\ Extracellular region}

Intracellular region

Occurrences

Motor activity

Ligase activity

Signal transducer activity

Oxiodoreductase activity

Isomerase activity

Enzyme regulator activity

Catalytic activity

Transferase activity

Structural molecule activity

Transporter activity

Occurrences
52
15
15
132

0

3

4

6

24

1

8

36

113

22

18

12

Occurrences

Cellular membrane fusion

3

Response to stimulus

18

0

26

Cell differentiation

17

Metabolic process

87

Regulation of biological process

52

Extracellular structure organization

0

Multicellular organismal development

23

Cellular process

40

Cell communication

3

3

Nucleobase, nucleoside, nucleotide and nucleic acid metabolic process found 30 statistically significant differentially expressed transcripts in our larval microarray experiments. MercadoCuriel and colleagues measured the temporal response of gene expression in adult male $R$. microplus in response to Anaplasma marginale infection [21]. When they compared infected ticks with uninfected controls using microarray assays, they determined that 888 genes were differentially expressed in midgut tissue 2 days post-infection and 146 genes were differentially expressed in the salivary glands 9 days post-infection. In contrast, Ribeiro and colleagues found only 10 differentially expressed genes in the salivary glands of Ixodes scapularis nymphs in response to Borrelia burgdorferi infection [22]. Rodriguez-Valle et al. found over 300 differentially expressed BmiGI transcripts in their study of $R$. microplus feeding upon Bos indicus and Bos taurus cattle [23]. Feeding upon tick resistant cattle as opposed to tick susceptible cattle evidently creates greater perturbations compared with feeding upon B. bovis-infected blood as opposed to uninfected cattle blood. Saldivar et al. discovered 76, 32, 80, and 83 differentially expressed BmiGI Version 2 transcripts in their microarray analysis of gene expression changes in response to larval exposure to the acaricides: 
coumaphos, permethrin, ivermectin and amitraz, respectively [4].

\section{Subtracted library results and discussion}

ESTs from the B. bovis-infected larval subtracted libraries were assembled and annotated. Additional file 1 contains data pertaining to annotated unigenes. Of the 791 total transcripts sequenced, 30 were classified as coming from B. bovis and removed from analysis. Three additional sequences, one from a mitochondrial gene, one rRNA sequence, and one putative transcript from Bos Taurus were also removed. Of the remaining 758 sequences, 193 had little sequence similarity to BmiGI Version 2 transcripts and therefore appear to be novel. The Uniref100 database searches revealed 381 sequences with similarity to known proteins.

\section{GO annotations}

The annot8r application provides a synopsis of the GO annotation process by categorizing the unigenes into 3 domains consisting of 29 high-level GO terms (Table 4). In the cellular component domain (C), most of the differentially expressed transcripts were deemed intracellular. The predominant annotation in the molecular function domain (F) was protein binding followed by catalytic activity and oxidoreductase activity. Metabolic processes were the largest component of the biological process domain $(\mathrm{P})$.

We further utilized the GO annotations to find unigenes with possible roles in the infection process $[24,25]$. Gene products identified via BLASTX searches with GO annotations of defense response (GO:000692) or stress response (GO:006950) are listed in Tables 5 and 6, respectively. Each unique annotation comprises the study sequence identifier, the source (database) of the annotation, a description of the annotation, the genus and species of the HSP, the NCBI accession number of the protein, and the corresponding e-value for the HSP.

Annotations for 6 unigenes from the assembled subtracted library sequencing were related to defense response by similarity to proteins in the Uniprot database annotated by GO (Table 5). Among the 6 ESTs, 2 were homologous to proteins in Ixodus scapularis. Unigene 402 showed significant similarity to 3-alpha-1-fucosyltransferase, which has been demonstrated to increase microbial pathogenesis in I. scapularis [26]. Unigene 468 was identified as a putative defense protein precursor by similarity to a protein from Bombyx mori. Two defense related hydrolases were also identified. Valacyclovir hydrolase (unigene 79) is designated as "response to toxin" by the GO ontology (GO:0009636). In addition, Bleomycin hydrolase (unigene 637) inactivates bleomycin B2 (a cytotoxic glycometallopeptide: GO:0009636). Unigene 113 displayed similarity to an Interferon gamma-inducible protein that in humans has a role in antigen processing and epitope presentation [27], but in ticks may upregulate cathepsins that activate serine proteases as an immune response. A putative salivary lipocalin (unigene 45) suggests a role in the tick's immune response since lipocalins are involved in many inflammation and detoxification processes in mammals [28]. Unigene 673 is a putative FYN binding protein; the GO Ontology annotates this protein with the term "defense response". As determined by BLAST search similarity to the KEGG database, Unigene 92 exhibited strong homology $(e$-value $=5 \mathrm{e}-176)$ to an $I$. scapularis protein within the Toll-like receptor-signaling pathway-an important component of the host innate immune system.

Annotations for 9 unigenes from the assembled subtracted library sequencing subsumed the GO term "response to stress" (GO:0006950) by similarity to proteins in the Uniprot database (Table 6). Moreover, 6 unigenes were homologous to proteins in I. scapularis. Unigenes 244 and 489 showed significant similarity to two I. scapularis cytochrome $\mathrm{P} 450$ proteins, which are potent detoxifiers of xenobiotics. Other observed detoxification proteins include: thioredoxin (unigene 416) and glutathione peroxidase (unigene 40), which facilitate the reduction of proteins and lipids, respectively. Two putative antioxidant peroxidase-related proteins (unigenes 89 and 187) were also identified. Two putative stress responserelated hydrolases were detected in the subtraction library. Valacyclovir hydrolase (unigene 79) is annotated with the biological process "response to toxin"

Table 5 Unigenes from subtracted library with BLAST database GO annotation of defense response (G0:0006952)

\begin{tabular}{|c|c|c|c|c|c|}
\hline \multirow[t]{2}{*}{ 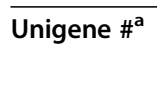 } & \multirow[t]{2}{*}{ Database } & \multicolumn{4}{|l|}{ BLASTX Annotation } \\
\hline & & Protein Annotation & Species & Accession Number & $e$-Value \\
\hline 45 & Uniref100 & Salivary lipocalin & Amblyomma variegatum & DAA34698.1 & $2 e-07$ \\
\hline 92 & KEGG & Toll-like receptor signaling pathway & Ixodes scapularis & XP002404081.1 & $5 e-176$ \\
\hline 113 & Uniref100 & Interferon gamma-inducible protein & Amblyomma americanum & AAK82985.1 & $2 e-92$ \\
\hline 402 & Uniref100 & Glycoprotein 3-alpha-I-fucosyltransferase A & Ixodes scapularis & XP002413615.1 & $3 e-70$ \\
\hline 468 & Uniref100 & Putative defense protein precursor & Bombyx mori & NP001091819.1 & $2 e-20$ \\
\hline 673 & Uniref100 & FYN binding protein & Ixodes scapularis & XР002412475.1 & $1 e-22$ \\
\hline
\end{tabular}

${ }^{a}$ Unigene identification number as listed in Additional file 1. 
Table 6 Unigenes from subtracted library with BLAST database GO annotation of stress response (G0:0006950)

\begin{tabular}{|c|c|c|c|c|c|}
\hline \multirow[b]{2}{*}{ Unigene $\#^{\mathrm{a}}$} & \multirow[b]{2}{*}{ Database } & \multicolumn{4}{|l|}{ BLASTX Annotation } \\
\hline & & Protein Annotation & Species & Accession Number & $e$-Value \\
\hline 40 & Uniref100 & Glutathione Peroxidase & Ixodes scapularis & XP002399259.1 & $1 e-41$ \\
\hline 79 & Uniref100 & Valacyclovir hydrolase & Ixodes scapularis & XP002404467.1 & $2 e-56$ \\
\hline 89 & $\mathrm{GO}$ & Hydrogen peroxide catabolism & Homo sapiens & A1KZ92 & $1 e-11$ \\
\hline 187 & Enzyme class & Peroxidase & Caenorhabditis elegans & Q1EN18 & $4 e-10$ \\
\hline 215 & Uniref100 & Heat shock protein & Locusta migratoria & AAO21473.1 & $4 \mathrm{e}-10$ \\
\hline 244 & Uniref100 & Cytochrome P450 & Ixodes scapularis & B7PSW2 & $1 e-81$ \\
\hline 416 & Uniref100 & Thioredoxin domain-containing protein & Ixodes scapularis & XP002436084.1 & $9 e-28$ \\
\hline 489 & Uniref100 & Cytochrome P450 & Ixodes scapularis & XP002414034.1 & $2 e-44$ \\
\hline 637 & Uniref100 & Bleomycin hydrolase & Ixodes scapularis & XP002399259.1 & $1 e-78$ \\
\hline
\end{tabular}

a Unigene identification number as listed in Additional file 1.

(GO:0009636). In addition, Bleomycin hydrolase (unigene 637) inactivates bleomycin B2 (a cytotoxic glycometallopeptide). Unigene 215 is homologous to a putative heat shock protein discovered in Locusta migratoria. Heat shock protein expression levels are generally upregulated when the organism is stressed.

The InterProScan algorithms yielded an additional 190 unique sequence annotations, which brought the total number of annotated ESTs to 571 out of 758 (75\%). No unigenes were annotated with the term "immune response" (GO:006950) by homology searches. However, Table 7 lists unigenes associated with immune function identified by InterProScan. Each unique annotation comprises the study sequence identifier, the protein, source (method) of the annotation, and the InterPro identifier assigned to this protein. InterProScan annotated 11 unigenes with immune or defense responserelated function including an additional lipocalin (unigene 443) that was not identified in the BLAST searches. Six of these unigenes were classified as immunoglobulin or immunoglobulin-like proteins. Since the tick has no adaptive immune system, we speculate that these proteins are either false positives or may serve a similar role to proteins that have homologs in vertebrates. For example, the human Down Syndrome cell adhesion molecule (DSCAM) has an immunoglobulin-like domain with several known homologs in arthropods that may have thousands of splice variants and help drive the clearance of pathogens $[29,30]$.

The remaining three unigenes were characterized as serine protease inhibitors (serpins). Recent interest in tick serpins stems from their significant antimicrobial and antifungal activity [31-33]. Serpins are cysteine-rich antimicrobial peptides and are components of the immune system of many invertebrates. Serpins have been used as target antigens for recombinant tick vaccines [34]. Unigenes 298 and 520 had sequence similarity (HSPs) to proteins that belong to the Trypsin inhibitor like cysteine rich domain (TIL) family. Huang et al. characterized a protein, C/E1 from Ascaris suum, with a

Table 7 Unigenes from subtracted library with InterProScan annotation of immune response

\begin{tabular}{llll}
\hline & InterProScan Annotation & & \\
\cline { 2 - 4 } Unigene $^{\mathbf{a}}$ & Protein Annotation & Methods & Interpro ID \\
\hline 298 & Serine Protease Inhibitor & HMMPfam, superfamily & IPR002919 \\
371 & Serine Protease Inhibitor & HMMPfam, superfamily & IPR002919 \\
520 & Serine Protease Inhibitor & HMMPfam, superfamily & IPR002919 \\
89 & Immunoglobulin-like & HMMPfam, HMMSmart, Gene3D, ProfileScan & IPR007110 \\
187 & Immunoglobulin-like & HMMPfam, HMMSmart, Gene3D, ProfileScan & IPR002919 \\
216 & Immunoglobulin & Superfamily & IPR013783 \\
288 & Immunoglobulin-like fold & HMMPfam, HMMSmart, Gene3D, ProfileScan & IPR002919 \\
520 & Immunoglobulin-like & HMMPfam, HMMSmart, Gene3D, ProfileScan & IPR007110 \\
601 & Immunoglobulin-like & HMMPfam, HMMSmart, ProfileScan, Superfamily & IPR008985 \\
242 & Lectin/glucanase & Gene3D, Superfamily & IPR011038 \\
\hline 433 & Lipocalin & Superfamily &
\end{tabular}

${ }^{a}$ Unigene identification number as listed in Additional file 1. 


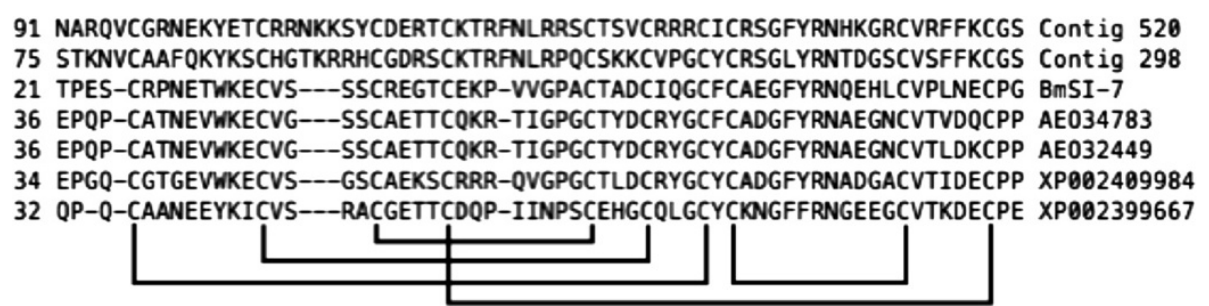

Figure 1 Multiple sequence alignment of two putative serpins identified in the subtracted larval library with a known serpin from $R$. microplus (BmSI-7), two putative serpins from Amblyomma maculatum (AEO34783 and AEO32449) and two putative serpins from Ixodes scapularis (XP002409984 and XP002399667). The five disulfide bridges formed by 5 pairs of cysteine residues are indicated by the black lines. The numbers to the left are the position of the first amino acid (first column) within each polypeptide.

TIL domain about 60 residues long containing five disulfide bonds - two of which are located on either side of the reactive site. Serpins with this domain were subsequently isolated and verified in $R$. microplus [35]. Figure 1 shows a multiple sequence alignment of unigenes 298 and 520 with a validated serpin from $R$. microplus (BmSI-7), two putative serpins from Amblyomma maculatum (AEO34783 and AEO32449) and two putative serpins from Ixodes scapularis (XP002409984 and XP002399667). The figure demonstrates that the portions of each protein containing the TIL domain, including the 5 cysteine residue pairs that form the disulfide bridges, are well aligned. Note that these protein domains were predicted by InterProScan and not found in the BLASTX search of the Uniprot100 database, thus underscoring the utility of de novo domain prediction algorithms.

Other noteworthy genes include three novel unigenes involved in signal transduction pathways. Unigene 192 was predicted to be low-density lipoprotein receptorrelated by InterProscan. The GO annotations included the term extracellular region (GO:0005576), hemoglobin import (GO:0020028) and lipoprotein transport (GO:0042954). This protein likely performs some binding function involved in the uptake of lipoproteins related to feeding on blood. Unigene 331 is a predicted membrane protein by InterProScan that is involved in ion transport. The BLAST annotation characterizes the protein as a voltage sensitive phosphatase and therefore may be involved in neural signal transduction. Unigene 412 is a putative porcupine-like protein that is integral to the Wnt signaling pathway. InterProScan predicted transmembrane regions in this protein including a signal peptide, which is further evidence of its relationship to membrane signal transduction.

\section{PCR results}

To verify the microarray results and the subtraction library results, we selected 2 transcripts from each result data set with significant differential expression of varying fold-changes and performed qRT-PCR to compare transcript levels in the B. bovis infected larval tissue to the uninfected larval controls. Table 8 shows that the directional expression changes for the selected transcripts were qualitatively similar in both the microarrays and qRT-PCRs. Table 9 likewise indicates that directional expression changes for the selected transcripts were qualitatively similar in both the subtracted library and qRT-PCRs.

\section{Conclusion}

In summary, our experimental approaches yielded specific differential gene expression associated with the infection of $R$. microplus larvae by $B$. bovis. However, the number of statistically significant differentially expressed transcripts was lower than we had anticipated. Microarray experiments determined that 24 transcripts were expressed in larval tissues at a statistically significant higher level in ticks feeding upon a B. bovis-infected calf contrasted to ticks feeding on an uninfected calf. Six transcripts were expressed in larval tissues at a statistically significant lower level in ticks feeding upon a B. bovis-infected calf contrasted to ticks feeding on an uninfected calf. Although the BmiGI Version 2 gene index was a useful database to help assign putative function to specific transcripts, a significant percentage of the differentially expressed transcripts did not have annotation that was useful for assignment of function. This emphasizes the need for further investigation of the genome of the cattle tick to develop a resource containing the full transcriptome. Our database of differentially expressed genes responding to $B$. bovis infection will be used to guide further investigations of the B. bovis-R. microplus complex.

Table 8 RT-PCR verification of selected microarray results

\begin{tabular}{llllll}
\hline EST & Microarray & & & \multicolumn{2}{l}{ Relative Quantitative PCR } \\
\cline { 2 - 3 } \cline { 5 - 6 } & Uninfected & Infected & & Uninfected & Infected \\
\hline TC12256 & 1.0 & 7.6 & & 1.0 & 8.4 \\
TC14244 & 3.4 & 1.0 & & 1.4 & 1.0 \\
\hline
\end{tabular}

${ }^{a}$ Normalized data to set lower value to 1 for comparison purposes. 
Table 9 RT-PCR verification of selected subtraction library results

\begin{tabular}{lll}
\hline Unigene $\#^{\mathbf{b}}$ & \multicolumn{2}{l}{ Relative Quantitative PCR } \\
\cline { 2 - 3 } & Uninfected & Infected \\
\hline 298 & 1.0 & 1.9 \\
520 & 2.1 & 1.0 \\
\hline
\end{tabular}

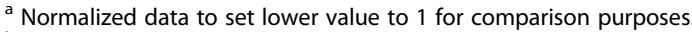

${ }^{\mathrm{b}}$ Unigene identification number as listed in Additional file 1.

The diversity of our approaches provides a good database of transcripts that express differential regulation in response to B. bovis infection. The ESTs from the subtracted libraries add to the list of genes involved in the tick infection process and we focused on transcripts related to the ticks' stress response and innate immune response. The functions of these transcripts might provide insight into the infection and transmission processes of $B$. bovis as it interacts with its host tick, $R$. microplus.

The subtraction library yielded 28 unique transcripts related to immune, defense, and stress responses, which implies an up-regulation of expression levels of the corresponding genes in response to $B$. bovis infection. It is also lends insight into the defense mechanisms at the disposal of $R$. microplus while still in the larval stage of development.

\section{Additional files}

\section{Additional file 1: ESTs from the Babesia bovis-infected larvae} subtracted library. This Excel file contains EST sequences from the subtracted library synthesized from the Babesia bovis-infected larvae (using uninfected larvae for the subtraction) and BLASTX annotation information including: GO, EC, KEGG, and InterProScan generated annotations.

Additional file 2: BmiGI entries significantly up- or down-regulated in microarray experiments. Analysis of microarray data resulted in identification of specific entries from the Rhipicephalus microplus gene index, BmiGl Version 2, that are statistically up- or down-regulated in response to Babesia bovis infection. This Excel file contains BmiGI ID number, hit descriptions, annotation, GO Terms, e-values, reading frames, and related information.

\section{Competing interests}

The authors declare that there are no competing interests.

\section{Authors' contributions}

FDG conceived the study, participated in the design, data collection, and analysis of the study and drafted the manuscript. AMH performed bioinformatic analysis of the ESTs and drafted the manuscript. KGB participated in the data collection, data analysis, and designed the RT-PCR verification study. LS and CG participated in analysis of the microarray data; GAS participated in the overall study design, infection of cattle, timing of tick infections and collection of tick materials. VN, SED and AD participated in study design and coordinated the sequencing phases. KAB participated in study design and microarray experimental design. All authors read and approved the final manuscript.

\section{Acknowledgements}

L.S. was supported by the National Research Initiative of the USDA CSREES grant \#2005-35604-15440 (to K.A.B. and F.D.G.). This article reports the results of research only. Mention of trade names or commercial products in this publication is solely for the purpose of providing specific information and does not imply recommendation of endorsement by the U.S. Department of Agriculture. USDA is an equal opportunity provider and employer.

\section{Author details}

'USDA-ARS, Knipling Bushland US Livestock Insect Research Laboratory, 2700 Fredericksburg Rd, Kerrville, TX 78028, USA. ${ }^{2}$ Department of Mathematics, University of Texas at El Paso, El Paso, TX 79968, USA. ${ }^{3}$ USDA-ARS Animal Disease Research Unit, Pullman, WA 99164, USA. ${ }^{4}$ The Institute for Genetics and Bioinformatics, University of New England, Armidale, NSW 2351, Australia. ${ }^{5}$ International Livestock Research Institute (ILRI) and Biosciences eastern and central Africa (BecA) Hub, PO Box 30709, Nairobi, Kenya. ${ }^{6}$ Program in Vector-Borne Diseases, Department of Veterinary Microbiology and Pathology, Washington State University, Pullman, WA 99164, USA.

Received: 24 May 2012 Accepted: 26 July 2012

Published: 7 August 2012

\section{References}

1. Grisi L, Massard CL, Moya Borja GE, Pereira JB: Impacto economico das principais ectoparasitoses em bovinos no Brasil. Hora Veterinaria 2002, 125:8-10.

2. de Castro JJ: Sustainable tick and tickborne disease control in livestock improvement in developing countries. Vet Parasitol 1997, 71:77-97.

3. Bock R, Jackson L, de Vos A, Jorgensen W: Babesiosis of cattle. Parasitol 2004, 129:S247-S269.

4. Saldivar L, Guerrero FD, Miller RJ, Bendele KG, Gondro C, Brayton KA: Microarray analysis of acaricide-inducible gene expression in the southern cattle tick, Rhipicephalus (Boophilus) microplus. Insect Molec Biol 2008, 17:597-606.

5. Sambrook J, Fritsch EF, Maniatis T: Molecular Cloning A Laboratory Manual. Cold Spring Harbor, New York: Cold Spring Harbor Laboratory Press; 1989.

6. Wasmuth JD, Blaxter ML: prot4EST: Translating Expressed Sequence Tags from neglected genomes. BMC Bioinforma 2004, 5:187.

7. Suzek BE, Huang H, McGarvey P, Mazumder R, Wu CH: UniRef: comprehensive and non-redundant UniProt reference clusters. Bioinformatics 2007, 23:1282-1288.

8. Altschul SF, Gish W, Miller W, Myers EW, Lipman DJ: Basic local alignment search tool. J Mol Biol 1990, 215:403-410.

9. Schmid R, Blaxter ML, annot8r: GO, EC and KEGG annotation of EST datasets. BMC Bioinforma 2008, 9:180.

10. Bairoch A: The ENZYME database in 2000. Nucleic Acids Res 2000, 28:304-305.

11. Kanehisa M, Goto S, Kawashima S, Nakaya A: The KEGG databases at GenomeNet. Nucleic Acids Res 2002, 30:42-46.

12. Mulder N, Apweiler R: InterPro and InterProScan: tools for protein sequence classification and comparison. Methods Mol Biol 2007, 396:59-70.

13. R Development Core Team. R: A language and environment for statistical computing. Vienna, Austria: R Foundation for Statistical Computing; 2009.

14. Gentleman RC, Carey VJ, Bates DM, Bolstad B, Dettling M, Dudoit S, Ellis B, Gautier L, Ge YC, Gentry J, Hornik K, Hothorn T, Huber W, lacus S, Irizarry R, Leisch F, Li C, Maechler M, Rossini AJ, Sawitzki G, Smith C, Smyth G, Tierney $L$, Yang JYH, Zhang JH: Bioconductor: open software development for computational biology and bioinformatics. Genome Biol 2004, 5:R80.

15. Irizarry RA, Bolstad BM, Collin F, Cope LM, Hobbs B, Speed TP: Summaries of affymetrix GeneChip probe level data. Nucleic Acids Res 2003, 31:e15.

16. Irizarry RA, Hobbs B, Collin F, Beazer-Barclay YD, Antonellis KJ, Scherf U, Speed TP: Exploration, normalization, and summaries of high density oligonucleotide array probe level data. Biostatistics 2003, 4:249-264.

17. Storey JD: A direct approach to false discovery rates. J R Stat Soc Ser B Stat Methodol 2002, 64:479-498.

18. Tusher VG, Tibshirani R, Chu G: Significance analysis of microarrays applied to the ionizing radiation response. Proc Natl Acad Sci USA 2001, 98:5116-5121.

19. Mulenga A, Macaluso KR, Simser JA, Azad AF: Dynamics of Rickettsia-tick interactions: identification and characterization of differentially expressed mRNAs in uninfected and infected Dermacentor variabilis. Insect Molec Biol 2003, 12:185-193.

20. Dreher-Lesnick SM, Mulenga A, Simser JA, Azad AF: Differential expression of two glutathione S-transferases identified from the American dog tick, Dermacentor variabilis. Insect Molec Biol 2006, 15:445-453. 
21. Mercado-Curiel RF, Palmer GH, Guerrero FD, Brayton KA: Temporal characterization of the organ-specific Rhipicephalus microplus transcriptional response to Anaplasma marginale infection. Int J Parasitol 2011, 41:851-860.

22. Ribeiro JMC, Alarcon-Chaidez F, Francischetti IMB, Mans BJ, Mather TN, Valenzuela JG, Wikel SK: An annotated catalog of salivary gland transcripts from Ixodes scapularis ticks. Insect Biochem Molec Biol 2006, 36:111-129.

23. Rodriguez-Valle M, Lew-Tabor A, Gondro C, Moolhuijzen P, Vance M, Guerrero FD, Bellgard M, Jorgensen W: Comparative microarray analysis of Rhipicephalus (Boophilus) microplus expression profiles of larvae preattachment and feeding adult female stages on Bos indicus and Bos taurus cattle. BMC Genomics 2010, 11:437.

24. Ashburner M, Ball CA, Blake JA, Botstein D, Butler H, Cherry JM, Davis AP, Dolinski K, Eppig JT, Harris MA, Hill DP, Issel-Traver L, Kasarskis A, Lewis S, Matese JC, Richardson JE, Ringwald M, Rubin GM, Sherlock G: Gene ontology: tool for the unification of biology. The Gene Ontology consortium. Nat Genet 2000, 25:25-29.

25. Camon E, Magrane M, Barrell D, Lee V, Dimmer E, Maslen J, Binns D, Harte N, Lopez R, Apweiler R: The Gene Ontology Annotation (GOA) Database: sharing knowledge in Uniprot with Gene Ontology. Nucleic Acids Res 2004, 32:D262-D266.

26. Pedra JHF, Narasimhan S, Rendic D, DePonte K, Bell-Sakyi L, Wilson IBH, Fikrig E: Fucosylation enhances colonization of ticks by Anaplasma phagocytophilum. Cellular Microbioology 2010, 12:1222-1234.

27. Goldstein OG, Hajiaghamohseni LM, Amria S, Sundaram K, Reddy SV, Haque A: Gamma-IFN-inducible-lysosomal thiol reductase modulates acidic proteases and HLS class II antigen processing in melanoma. Cancer Immunol Immunother 2008, 57:1461-1470.

28. Flower DR, Attwood TK, North AC: The lipocalin protein family: structure and function. Biochem J 1996, 318:1-14

29. Dong YM, Taylor HE, Dimopoulos G: AgDscam, a hypervariable immunoglobulin domain-containing receptor of the Anopheles gambiae innate immune system. Plos Biology 2006, 4:1137-1146.

30. Watson FL, Puttmann-Holgado R, Thomas F, Lamar DL, Hughes M, Kondo M, Rebel VI, Schmucker D: Extensive diversity of Ig-superfamily proteins in the immune system of insects. Science 2005, 309:1874-1878.

31. Fogaça AC, Lorenzini DM, Kaku LM, Esteves E, Bulet P, Daffre S: Cysteinerich antimicrobial peptides of the cattle tick Boophilus microplus: isolation, structural characterization and tissue expression profile. Develop Comp Immunol 2004, 28:191-200.

32. Gronenborn AM, Nilges M, Peanasky RJ, Clore GM: Sequential resonance assignment and secondary structure determination of the Ascaris trypsin inhibitor, a member of a novel class of proteinase inhibitors. Biochemistry 1990, 29:183-189.

33. Silva FD, Rezende CA, Rossi DC, Esteves E, Dyszy FH, Schreier S, Gueiros-Filho F, Campos CB, Pires JR, Daffre S: Structure and mode of action of microplusin, a copper II-chelating antimicrobial peptide from the cattle tick Rhipicephalus (Boophilus) microplus. J Biol Chem 2009, 284:34735-34746.

34. Sugino M, Imamura S, Mulenga A, Nakajima M, Tsuda A, Ohashi K: A serine proteinase inhibitor (serpin) from ixodid tick Haemaphysalis longicornis; cloning and preliminary assessment of its suitability as a candidate for a tick vaccine. Vaccine 2003, 21:2844-2851.

35. Sasaki SD, de Lima CA, Lovato DV, Juliano MA, Torquato JS, Tanaka AS: BmSI-7, a novel subtilisin inhibitor from Boophilus microplus, with activity toward Pr1 proteases from the fungus Metarhizium anisopliae. Exp Parasitol 2008, 118:214-220.

doi:10.1186/1756-3305-5-162

Cite this article as: Heekin et al:: Analysis of Babesia bovis infectioninduced gene expression changes in larvae from the cattle tick, Rhipicephalus (Boophilus) microplus. Parasites \& Vectors 2012 5:162

\section{Submit your next manuscript to BioMed Central and take full advantage of:}

- Convenient online submission

- Thorough peer review

- No space constraints or color figure charges

- Immediate publication on acceptance

- Inclusion in PubMed, CAS, Scopus and Google Scholar

- Research which is freely available for redistribution

Submit your manuscript at www.biomedcentral.com/submit
C BioMed Central 